\title{
A novel frameshift mutation of PRRT2 in a family with infantile convulsions and choreoathetosis syndrome: c.640delinsCC (p.Ala214ProfsTer11)
}

\author{
Bo Mi Park', Young Ok Kim*, Myeong-Kyu Kim², and Young Jong Woo \\ ${ }^{1}$ Department of Pediatrics, Chonnam National University Medical School, Gwangju, Korea \\ ${ }^{2}$ Department of Neurology, Chonnam National University Medical School, Gwangju, Korea
}

The infantile convulsions and choreoathetosis (ICCA) syndrome is defined when two overlapping clinical features of benign familial infantile epilepsy (BFIE) and paroxysmal kinesigenic dyskinesia (PKD) are present in an individual or a family. Since the gene encoding proline-rich transmembrane protein 2 (PRRT2) was first identified in Han Chinese families with PKD, mutations of PRRT2 have additionally been reported in patients with BFIE and ICCA. We attempted to identify the genetic etiology in an ICCA family where the proband, her elder sister, and a maternal male cousin had BFIE, and her mother had PKD. Whole-exome sequencing performed in the proband and her sister and mother identified a novel pathogenic mutation of PRRT2 (c.640delinsCC; p.Ala214ProfsTer11), which was verified by Sanger sequencing. This frameshift PRRT2 mutation located near the genetic hot spot of base 649_650 results in the premature termination of the protein, as do most previously reported mutations in BFIE, ICCA, and PKD.

Key words: PRRT2, Seizures, Dyskinesias, Family.

\section{Introduction}

The infantile convulsions and choreoathetosis (ICCA) syndrome is a rare overlapping syndrome of epilepsy and movement disorder involving clinical features of benign familial infantile epilepsy (BFIE) and paroxysmal kinesigenic dyskinesia (PKD) with an autosomal dominant inheritance. BFIE and PKD can occur in different members of the same family or can cooccur in the same patient [1-6]. Linkage analyses of families with ICCA, BFIE, or PKD have revealed linkage in a pericentromeric region of chromosome $16[1,2]$.

$\mathrm{BFIE}$ is an epilepsy disorder characterized by infantile-onset
(4 to 12 months of age) self-limiting seizures (before 2 years of age) [1-6]. The seizures are usually focal nonmotor ones that occur in clusters, and they are easily controlled by antiepileptic drugs [1-6]. Infants with BFIE exhibit normal development [1-7]. PKD is a late-childhood-onset movement disorder that presents with brief and recurrent involuntary movements (e.g., dystonia, chorea, athetosis, and ballism) without altered consciousness [6,8-10]. Dyskinetic movements that are provoked by voluntarily performing sudden actions from rest (e.g., starting to stand, walk, or run) respond well to certain antiepileptic drugs such as carbamazepine and phenytoin, and gradually decrease with age, eventually disappearing in adulthood [6,8-10].

Received: 26 March 2019, Revised: 29 April 2019, Accepted: 30 April 2019, Published: 30 June 2019

${ }^{*}$ Corresponding author: Young Ok Kim, M.D., Ph.D. iD https://orcid.org/0000-0002-7873-1140

Department of Pediatrics, Chonnam National University Children's Hospital, 42 Jebong-ro, Dong-gu, Gwangju 61469, Korea.

Tel: +82-62-220-6646, Fax: +82-62-222-6103, E-mail: ik052@jnu.ac.kr

Conflict of interest: The authors declare that they do not have any conflicts of interest.

(ac) This is an open-access article distributed under the terms of the Creative Commons Attribution Non-Commercial License (http://creativecommons.org/licenses/by-nc/4.0/) which permits unrestricted non-commercial use, distribution, and reproduction in any medium, provided the original work is properly cited.

(c) Copyright 2019 by the Korean Society of Medical Genetics and Genomics 
Mutations of the gene encoding proline-rich transmembrane protein 2 (PRRT2; PRRT2, NM_145239.2, on 16p11.2) were first identified in eight Han Chinese families with pure PKD in 2011 [8]. Wang et al. [9] reported an additional five PKD families with PRRT2 mutations in the same year, two of whom had ICCA. PRRT2 mutations have subsequently been reported in ICCA families, most of which were c.649_650insC (p.Arg217ProfsTer7) regardless of ethnicity $[3-6,9,10]$. In the present ICCA family we identified a novel pathogenic mutation of PRRT2 (c.640delinsCC; p.Ala214ProfsTer11), which is at the fragile nine polycytosine tract (poly-C) stretch including the genetic hot spot (c.649_650insC).

\section{Case}

\section{Patients}

The 5-month-old female proband visited our hospital due to focal nonmotor seizures without awareness that were associated with lip smacking and cyanosis. She had previously been healthy. She was born at a gestational age of 41 weeks weighing $3.67 \mathrm{~kg}$ after an uneventful pregnancy. Her father was healthy, while her mother had a past history of paroxysmal dystonic events when she was in her teens. Her dyskinetic events were provoked by sudden standing, walking or running. Her symptoms usually last for less than one minute and occurred two or three times daily. These paroxysmal movements presented when she was around 12 years of age, gradually resolved after 18 years of age and disappear in her third decade. Her symptoms were compatible with PKD. The elder sister and a maternal male cousin of the proband experienced similar focal nonmotor seizures without awareness when they were 5 to 6 months of age. Their brain magnetic resonance images (MRIs) and electroencephalograms (EEGs) were normal, and their seizures responded to carbamazepine. Based on their family history and normal development, BFIE was diagnosed. The elder sister of the proband was seizure-free after 15 months of age (Fig. 1).

An examination performed at the proband's first visit to our hospital indicated that she had developed normally: she could roll over, could transfer a large cube from hand to hand, could babble monosyllabic sounds, preferred her mother to other people, and enjoyed looking in a mirror. The findings of a neurological examination, neonatal screening test with tandem mass, brain MRI and EEG were all normal. The electrolyte levels in her serum and her blood glucose, ammonia, and lactate levels were all within the normal ranges. Her first seizure lasted for less than 5 minutes, and it was followed by postictal sleep. The second seizure with the same pattern appeared 3 hours later on the same day. She was diagnosed as having BFIE, as were her affected sister and male cousin. She had been taking carbamazepine (10 $\mathrm{mg} / \mathrm{kg} /$ day) for one year, and had experienced no more seizures even after stopping carbamazepine.

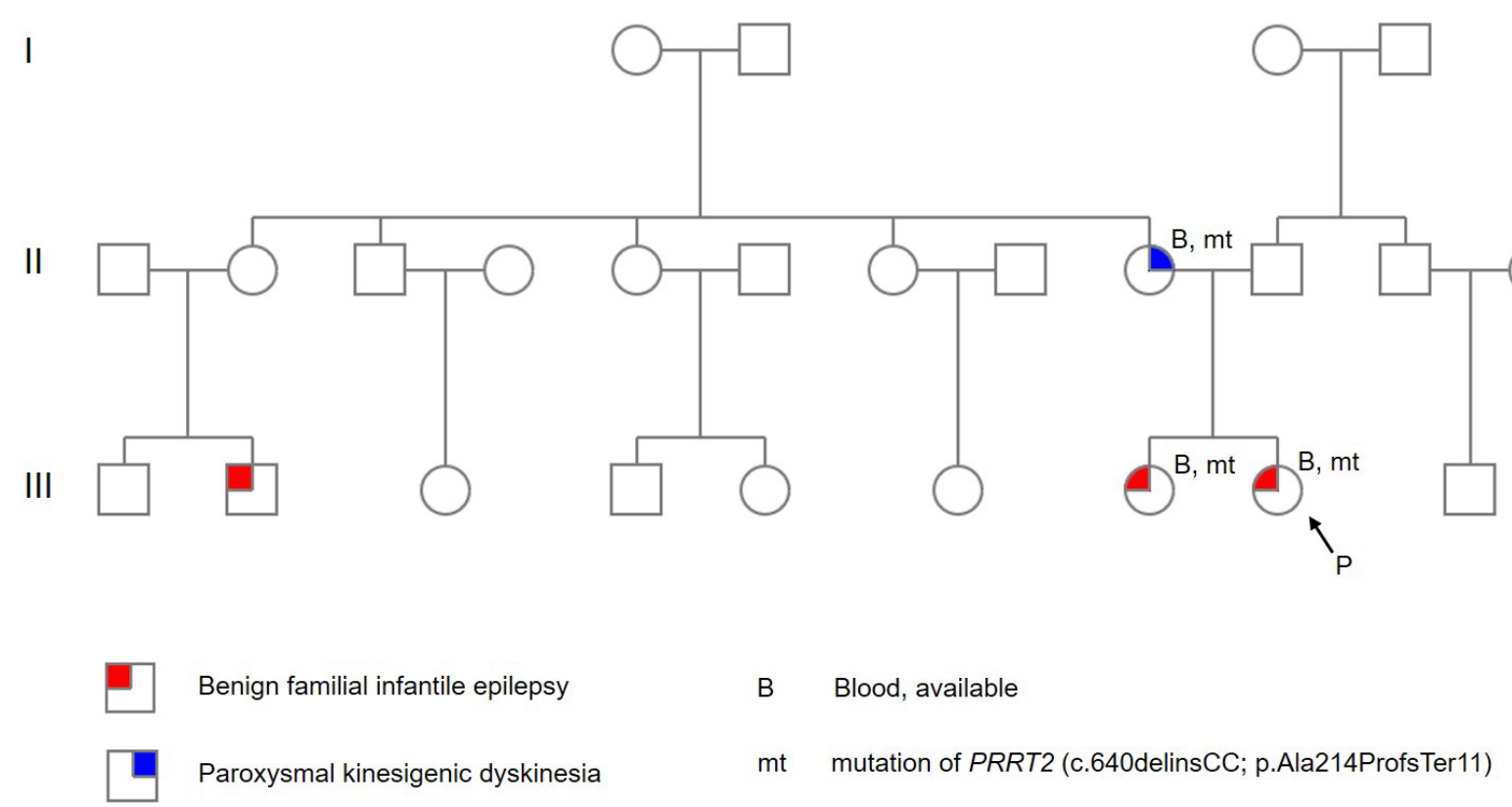

Fig. 1. Family pedigree in an infantile convulsions and choreoathetosis family. The proband, her elder sister, and a maternal male cousin had benign familial infantile epilepsy, and her mother had paroxysmal kinesigenic dyskinesia. Whole-exome sequencing and Sanger sequencing were performed in the proband and her sister and mother, which found the novel pathogenic variant of PRRT2. 


\section{Molecular genetic analysis}

Whole-exome sequencing (WES) was performed in the proband and her sister and mother using the TruSeq Exome Kit (Illumina Inc., San Diego, CA, USA) on the HiSeq2000 platform (Illumina Inc.). The variants were prioritized according to our bioinformatics workflow that considers possible inheritance. Various databases of single-nucleotide polymorphisms (SNPs) were referred to, including the Korean Reference Genome Database that includes 1,100 Korean controls (http://coda.nih.go.kr/ coda/KRGDB/index.jsp). The novel pathogenic variant of PRRT2 (NM_145239.2; c.640delinsCC; p.Ala214ProfsTer11) was found in the proband and her sister in an autosomal dominant model, but was not called from her mother. However, this variant on the starting site of a homopolymer tract of nine cytosines was verified by Sanger sequencing in all three of the affected family members (Fig. 2). The $\mathrm{G}$ on position 640 was deleted and CC were inserted resulting in 11 poly-C stretch. The novel heterozygous frameshift PRRT2 mutation was not found in any SNP databases, and it was a uniquely meaningful variant for ICCA in this family. A segregation study of the proband's biological father could not be performed due to the nonavailability of blood samples.
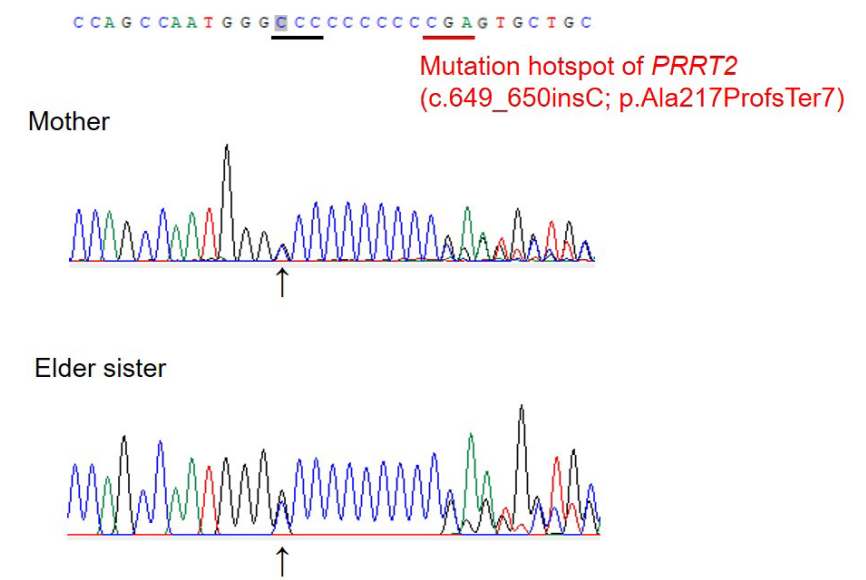

Proband

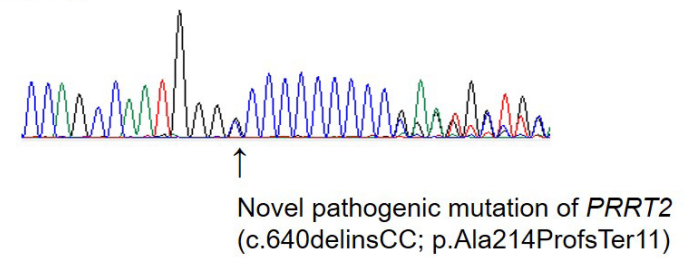

Fig. 2. Electropherogram showing the novel heterozygous frameshift mutation of PRRT2. Sanger sequencing in all three of the affected family members showed the novel pathogenic variant of PRRT2 (c.640delinsCC), which located near the genetic hot spot of base 649_650.
This study was reviewed and approved by the Human Research Ethics Committee of Chonnam National University Hospital (IRB no. CNUH-2017-167). Informed consent was obtained from the proband's mother. The biospecimens used in this study were provided by the Chonnam National University Hospital Biomedical Research Institute Biobank according to the protocols approved by the Institutinal Reveiw Board.

\section{Discussion}

PRRT2 consists of four exons, with the first one being noncoding [8]. PRRT2 protein is composed of 340 amino acids, with a proline-rich extracellular domain in the $\mathrm{N}$-terminal half and two transmembrane and one cytoplasmic domains in the C-terminal half $[6,8]$. Most pathogenic mutations of PRRT2 are frameshift, nonsense, and splice-site mutations that result in protein truncation, and most of these truncation mutations encode the extracellular domain of PRRT2 [3-10]. Missense mutations of PRRT2 occur in only a few patients, and are located around transmembrane domains [3-10]. Investigations of the relationships between the genotypes of PRRT2 and the phenotypes of ICCA, PKD, and BIFE have failed to reveal any clear correlations [6].

The genetic hot spot of PRRT2 mutations is base 649_650, which is at the end of a nine-poly- $C$ stretch forming a hairpin loop: c.649_650insC (p.Arg217ProfsTer8), c.649delC (p.Arg217GlufsTer12), and c.649C>T (p.Arg217Ter) [3-10]. All of these insertion, deletion, and nonsense mutations result in the premature truncation of PRRT2 [3-10]. The nine-polyC stretch is the unstable DNA site that is prone to breakage by polymerase slippage $[3,6,7]$. The insertion or deletion of bases on this poly-C stretch is less likely to be identified by WES, and can appear as 'a noisy sequence' even in direct sequencing as found in the studies of Heron, Schubert, Ono and their colleagues [3,5-7]. The novel pathogenic variant of PRRT2 (c.640delinsCC; p.Ala214ProfsTer11) that we found was not clearly called in the proband's mother by WES and the downstream sequence from the deletion of $\mathrm{G}$ and insertion of CC seemed ambiguous at first glance even in direct sequencing.

The frequencies of PRRT2 mutations in the patients with BFIE or PKD have been reported to differ between familial cases and sporadic cases [3-10]. According to Méneret et al's research [10] in a European population with PKD (including 2 ICCA families), PRRT2 mutations were found in 93\% (13 families) of 14 familial cases and in 45\% (9 patients) of 20 sporadic patients. In familial cases with ICCA, PKD, or BIFE, most of 
the PRRT2 mutations were the same frameshift mutation of c.649_650insC (p.Arg217ProfsTer8) [3-10]. This phenomenon has also been found in European, Oceanian, and Asian families [3-10]. Schubert et al. [7] recommended screening the sequence of c.649_650insC first and then performing full sequencing of PRRT2 in the identified noncarriers of c.649_650insC. In addition, the possibility of microdeletion encompassing PRRT2 has to be considered in patients with negative sequencing results [6]. PRRT2 is expressed strongly in the brain and spine tissues of mice $[3,8]$. Chen et al. [8] measured the expression levels of PRRT2 mRNA in murine brain lysates, and found that they were low in the embryonic period but increased after birth to peak at postnatal day 14 (P14) before dropping in adulthood. PRRT2 mRNA at P14 was predominantly detected in the cerebral cortex, hippocampus, and cerebellum [8]. Heron et al. [3] analyzed PRRT2 expression in the murine brain at P21 and P46, and reported that $P R R T 2$ was expressed strongly in the cerebral cortex but more weakly in the basal ganglia. In spatiotemporal transcriptome data of the human brain (data available at http:// hbatlas.org/), PRRT2 was expressed somewhat highly in the cerebellum, striatum, and neocortex, like in mice [11]. However, PRRT2 exon array signal intensity was markedly increased only before 12 or 19 post-conceptional weeks, and then stagnated in most brain regions other than the cerebellum (where it decreased and then gradually increased) and the thalamus (where it decreased markedly from the peak) [11].

The exact functional roles of PRRT2 mutations in the pathogeneses of ICCA, PKD, and BIFE remain unclear, with current hypotheses supported only by indirect evidence. Firstly, PRRT2 is expressed in the brain including the basal ganglia, where the expression levels seem to change with age in the murine brain $[3,8,11]$. Secondly, PRRT2 interacts with synaptosomal associated protein 25 , which is expressed strongly in the brain including the basal ganglia and is thought to regulate neurotransmitter release, since it is bound to a presynaptic plasma membrane and forms synaptic vesicles $[3,4,6,9]$. However, there is no precise explanation yet as to how a sodium-channel blocker is effective at improving the symptoms of patients with PRRT2 mutations given that these mutations seem to result in synaptopathy rather than channelopathy.

Here we have reported an ICCA family with a novel PRRT2 mutation that results in the premature termination of the protein (c.640delinsCC; p.Ala214ProfsTer11), which is at the start of a nine-poly-C stretch. This heterozygous frameshift PRRT2 mutation is located near the genetic hot spot of PRRT2 (c.649_650insC; p.Arg217ProfsTer7), which is at the end of the same poly-C stretch.

\section{Acknowledgements}

This research was supported by the Basic Science Research Program through the National Research Foundation of Korea (NRF) funded by the Ministry of Education, Republic of Korea (grant no. NRF-2017R1D1A3A03000532).

\section{References}

1. Szepetowski P, Rochette J, Berquin P, Piussan C, Lathrop GM, Monaco AP. Familial infantile convulsions and paroxysmal choreoathetosis: a new neurological syndrome linked to the pericentromeric region of human chromosome 16. Am J Hum Genet 1997;61:889-98.

2. Swoboda KJ, Soong B, McKenna C, Brunt ER, Litt M, Bale JF Jr, et al. Paroxysmal kinesigenic dyskinesia and infantile convulsions: clinical and linkage studies. Neurology 2000;55:224-30.

3. Heron SE, Grinton BE, Kivity S, Afawi Z, Zuberi SM, Hughes JN, et al. PRRT2 mutations cause benign familial infantile epilepsy and infantile convulsions with choreoathetosis syndrome. Am J Hum Genet 2012;90:152-60.

4. Lee HY, Huang $Y$, Bruneau $N$, Roll $P$, Roberson ED, Hermann $M$, et al. Mutations in the gene PRRT2 cause paroxysmal kinesigenic dyskinesia with infantile convulsions. Cell Rep 2012;1:2-12.

5. Ono S, Yoshiura K, Kinoshita A, Kikuchi T, Nakane Y, Kato N, et al. Mutations in PRRT2 responsible for paroxysmal kinesigenic dyskinesias also cause benign familial infantile convulsions. J Hum Genet 2012;57:338-41.

6. Heron SE, Dibbens LM. Role of PRRT2 in common paroxysmal neurological disorders: a gene with remarkable pleiotropy. J Med Genet 2013;50:133-9.

7. Schubert J, Paravidino R, Becker F, Berger A, Bebek N, Bianchi $A$, et al. PRRT2 mutations are the major cause of benign familial infantile seizures. Hum Mutat 2012;33:1439-43.

8. Chen WJ, Lin Y, Xiong ZQ, Wei W, Ni W, Tan GH, et al. Exome sequencing identifies truncating mutations in PRRT2 that cause paroxysmal kinesigenic dyskinesia. Nat Genet 2011;43:1252-5.

9. Wang JL, Cao L, Li XH, Hu ZM, Li JD, Zhang JG, et al. Identification of PRRT2 as the causative gene of paroxysmal kinesigenic dyskinesias. Brain 2011;134:3493-501.

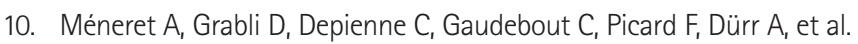
PRRT2 mutations: a major cause of paroxysmal kinesigenic dyskinesia in the European population. Neurology 2012;79:170-4.

11. Kang HJ, Kawasawa Yl, Cheng F, Zhu Y, Xu X, Li M, et al. Spatiotemporal transcriptome of the human brain. Nature 2011;478:483-9. 